\title{
Falling Dynamics of SARS-CoV-2 as a Function of Respiratory Droplet Size and Human Height
}

\author{
Mehmet Aydin $^{1}$ (D) Fatih Evrendilek ${ }^{2}$ (D) Seckin Aydin Savas ${ }^{3}$ (D) Ismail Erkan Aydin ${ }^{4}$ (D) Deniz Eren Evrendilek ${ }^{5}$ (D)
}

Received: 10 May 2020 / Accepted: 12 October 2020 / Published online: 19 October 2020

(c) Taiwanese Society of Biomedical Engineering 2020

\begin{abstract}
Purpose The purpose of this study is to quantify the motion dynamics of the severe acute respiratory syndrome coronavirus 2 (SARS-CoV-2).

Methods Three physical models of Newton's and Stokes's laws with(out) air resistance in the calm air are used to determine the falling time and velocity regimes of SARS-CoV-2 with(out) a respiratory water droplet of 1 to 2000 micrometers $(\mu \mathrm{m})$ in diameter of an infected person of 0.5 to $2.6 \mathrm{~m}$ in height.

Results The horizontal distance travelled by SARS-CoV-2 in free fall from $1.7 \mathrm{~m}$ was $0.88 \mathrm{~m}$ due to breathing or talking and $2.94 \mathrm{~m}$ due to sneezing or coughing. According to Newton's laws of motion with air resistance, its falling velocity and time from $1.7 \mathrm{~m}$ were estimated at $3.95 \times 10^{-2} \mathrm{~m} \mathrm{~s}^{-1}$ and $43 \mathrm{~s}$, respectively. Large droplets $>100 \mu \mathrm{m}$ reached the ground from $1.7 \mathrm{~m}$ in less than $1.6 \mathrm{~s}$, while the droplets $\geq 30 \mu \mathrm{m}$ fell within $4.42 \mathrm{~s}$ regardless of the human height. Based on Stokes's law, the falling time of the droplets encapsulating SARS-CoV-2 ranged from $4.26 \times 10^{-3}$ to $8.83 \times 10^{4} \mathrm{~s}$ as a function of the droplet size and height.

Conclusion The spread dynamics of the COVID-19 pandemic is closely coupled to the falling dynamics of SARS-CoV-2 for which Newton's and Stokes's laws appeared to be applicable mostly to the respiratory droplet size $\geq 237.5 \mu \mathrm{m}$ and $\leq 237.5 \mu \mathrm{m}$, respectively. An approach still remains to be desired so as to better quantify the motion of the nano-scale objects.
\end{abstract}

Keywords 2019-nCoV · SARS-CoV-2 · COVID-19 · Newton's laws · Stokes's law

Mehmet Aydin

ismailerkanaydin@odu.edu.tr

Fatih Evrendilek

fevrendilek@ibu.edu.tr

Seckin Aydin Savas

seckin.aydin@alanya.edu.tr

Ismail Erkan Aydin

ismailerkanaydin@odu.edu.tr

Deniz Eren Evrendilek

devrendi@sfu.ca

1 Emeritus, Department of Soil Science (Soil Physics), Mustafa Kemal University, Hatay, Turkey

2 Department of Environmental Engineering, Bolu Abant Izzet Baysal University, Bolu, Turkey

3 Department of Plastic and Reconstructive Surgery, Faculty of Medicine, Alanya Alaadin Keykubat University, Alanya/Antalya, Turkey

4 Department of Emergency Medicine, Faculty of Medicine, Ordu University, Ordu, Turkey

5 Department of Computing Science, Simon Fraser University, Burnaby, BC V5A 1S6, Canada

\section{Introduction}

The number of virus particles on the Earth has been recently estimated at close to $10^{31}$ [1]. The newly emergent 2019 novel coronavirus (2019-nCoV) disease (COVID-19), whose virus name has been changed by the International Committee on Taxonomy of Viruses to severe acute respiratory syndrome coronavirus 2 (SARS-CoV-2), has caused a global pandemic with serious impacts on the public and environmental health and human welfare. Therefore, it is vital to physically understand and control its spread mechanism. The size of viruses varies between 20 and 300 nanometers $(\mathrm{nm})$ in diameter $\left(1 \mathrm{~nm}=10^{-9} \mathrm{~m}\right)$ [2], while the size of SARSCoV-2 ranges from 60 to $140 \mathrm{~nm}$ in diameter [3]. This brief article aimed to explore three simple physical models to estimate the falling dynamics of SARS-CoV-2 that is transmitted through a respiratory droplet including coughing, sneezing, and expectorating of 1 to 2000 micrometers $(\mu \mathrm{m})$ in diameter of an infected person of 0.5 to $2.6 \mathrm{~m}$ in height. The novelty of this study stems from its first comparative 
quantification of the falling dynamics of SARS-CoV-2 as a function of respiratory droplet size and human height based on Newton's and Stokes's laws.

\section{Physical Models of Falling Dynamics of SARS-CoV-2}

In this study, SARS-CoV-2 was assumed as a single particle of a spherical shape with an average diameter of $100 \mathrm{~nm}$, radius of $5.0 \times 10^{-8} \mathrm{~m}$, and a protein density of $1.35 \times 10^{3} \mathrm{~kg} \mathrm{~m}^{-3}$. Whether be encapsulated in a respiratory droplet or not, the volume and mass of the virus can be estimated thus:

Volume $=4 / 3 \pi r^{3}$

$m=\rho p\left(4 / 3 \pi r^{3}\right)$

where $r, m$, and $\rho p$ are the particle radius (m), mass (kg), and density $\left(\mathrm{kg} \mathrm{m}^{-3}\right)$, respectively. Presented below are the three physical models, namely, one without air resistance (drag) and two with air resistance.

\subsection{A Free-Falling Particle Without Air Resistance}

In Newtonian physics, free fall is the simplest model where gravity is the only force that acts upon a falling particle. The vertical velocity in free fall is expressed as follows:

$v_{y}=g t=\sqrt{2 g h}$

$h=\frac{1}{2} g t^{2}$

$t=\sqrt{\frac{2 h}{g}}$

$X=V_{o} t=V_{o} \sqrt{\frac{2 h}{g}}$

where $v_{y}$ is the downward velocity $\left(\mathrm{m} \mathrm{s}^{-1}\right) ; g$ is the gravitational acceleration $\left(9.81 \mathrm{~m} \mathrm{~s}^{-2}\right) ; h$ is falling distance $(\mathrm{m}) ; t$ is falling time (s); $X$ is the horizontal distance (m); and $V_{o}$ is the initial velocity of the particle $\left(\mathrm{m} \mathrm{s}^{-1}\right)$.

\subsection{A Falling Particle with Air Resistance}

Not only is a falling particle through the atmosphere exposed to the gravitational force but also to air resistance. Newton's and Stokes's laws were used to estimate the falling dynamics of SARS-CoV-2. Based on Newton's law, the following two forces can be introduced:

$F g=m g$

$F a=\frac{\kappa \rho a A v^{2}}{2}$

where $F g$ is the downwards force due to gravity (Newton); $m$ is mass of the falling object $(\mathrm{kg}) ; F a$ is the air resistance (Newton) in the opposite direction to the velocity of the object; $\kappa$ is a shape-dependent coefficient $(0.47$ for a spherical particle); $\rho a$ is the air density $\left(1.2041 \mathrm{~kg} \mathrm{~m}^{-3}\right.$ at $20{ }^{\circ} \mathrm{C}$ and $1 \mathrm{~atm}) ; A$ is the cross-surface area of the object $\left(\mathrm{m}^{2}\right)$; and $v$ is the velocity of the particle $\left(\mathrm{m} \mathrm{s}^{-1}\right)$. The air resistance and gravitational forces can be combined in the following equation:

$m a=m g-\frac{\kappa \rho a A v^{2}}{2}$

where $m a$ is the net force in the falling/vertical direction according to the Newton's second law of motion (the net force is defined as the sum of all the forces acting on an object); and $a$ is the acceleration of the object $\left(\mathrm{m} \mathrm{s}^{-2}\right)$. This law applies mostly to the macro-scale objects flying with a high speed at the height of at least several meters. Nevertheless, this law may sufficiently reflect the motion of SARS$\mathrm{CoV}-2$ in the calm air since its initial velocity in the case of sneezing/coughing was reported to be $5.0 \mathrm{~m} \mathrm{~s}^{-1}$ [4]. Thus, Eq. (9) can be re-arranged as follows:

$a=g-\frac{\kappa \rho a A v^{2}}{2 m}$

Assuming that $a=0$ at the final or terminal velocity $\left(v_{t}\right.$, $\mathrm{m} \mathrm{s}^{-1}$ ) yields the following:

$g-\frac{\kappa \rho a A v_{t}^{2}}{2 m}=0$

$v_{t}=\sqrt{\frac{2 m g}{\kappa \rho_{a} A}}$

Since the acceleration is not constant, the air resistance near the end of the fall is greater than it is at the beginning of the fall. The time $(t)$ for the particles to fall down from a given height $(h)$ in the calm air can be approximated thus:

$t=h / v_{t}$

As was stated above, a falling particle in a fluid (the air in this study) encounters a frictional resistance proportional to the product of its radius and velocity and to 
the viscosity of the fluid. The resisting force $(\mathrm{Fa})$ due to friction was shown by Stokes in 1851 as follows [5]:

$F_{a}=6 \pi \eta r v$

where $\eta$ is the viscosity of the fluid, $1.85 \times 10^{-5} \mathrm{~kg} \mathrm{~m}^{-1} \mathrm{~s}^{-1}$, for the dynamical viscosity of air according to Anchordoqui and Chudnovsky [6]. According to Stokes's law, the terminal/constant velocity is estimated thus [5]:

$v_{t}=\frac{2 r^{2} g}{9 \eta}\left(\rho_{p}-\rho_{a}\right)=\frac{d^{2} g}{18 \eta}\left(\rho_{p}-\rho_{a}\right)$

where $d$ is the particle diameter $(\mathrm{m})$. A respiratory water droplet encapsulating SARS-CoV-2 can be assumed to have a density $\left(\rho_{w}\right)$ of 997 or $998 \mathrm{~kg} \mathrm{~m}^{-3}$ (for a droplet $\leq 1 \mu \mathrm{m}$ in diameter). The downward force on a spherical droplet due to gravity can be estimated neglecting the air density thus (please note that $\rho_{w} \approx 830 \rho_{a}$ ):

$F_{g} \cong \rho_{w}\left(4 / 3 \pi r^{3}\right) g$

Given the conditions of terminal velocity, the following Eqs. can be written:

$g-\frac{6 \pi \eta r v}{m}=0$

$v_{t}=\frac{m g}{6 \pi \eta r}$

Assuming that the terminal velocity is attained almost instantly, the falling time $(t)$ from a height $(h)$ can be expressed thus: $t=\frac{18 h \eta}{d^{2} g\left(\rho_{p}-\rho_{a}\right)}=\frac{h}{v_{t}}$

Using the relevant equations, the falling dynamics of a single isolated SARS-CoV-2 and a respiratory droplet encapsulating it through the atmosphere were simulated as a function of the human heights of 0.5 (the world's shortest person: $0.54 \mathrm{~m}$ ), $0.8,1.1,1.4,1.7,2.0,2.3$, and $2.6(\mathrm{~m})$ (the world's tallest person: $2.51 \mathrm{~m}$ ) and the respiratory droplet size range of 1 to $2000 \mu \mathrm{m}$ in diameter.

\section{Results and Discussion}

\subsection{The Motion of SARS-CoV-2 Without a Respiratory Droplet}

According to Eqs. (1) and (2), the volume and mass of a single SARS-CoV-2 were estimated at $5.236 \times 10^{-22} \mathrm{~m}^{3}$ and $7.07 \times 10^{-19} \mathrm{~kg}$, respectively. In free fall, the mass and shape do not affect the motion. According to Eq. (5), SARS-CoV-2 in free fall from $1.7 \mathrm{~m}$ (a typical human height adopted in medical procedures) was estimated to reach the ground in $0.589 \mathrm{~s}$ (Fig. 1a). In this case, the corresponding free-fall velocity $\left(5.775 \mathrm{~m} \mathrm{~s}^{-1}\right)$ is always greater than the real velocity $\left(2.888 \mathrm{~m} \mathrm{~s}^{-1}\right)$. However, from the physics point of view, a vacuum fall approach cannot be sufficiently justified for the falling velocity of SARS-CoV-2 through the atmosphere. Assuming the velocity of SARS-CoV-2 of $1.5 \mathrm{~m} \mathrm{~s}^{-1}$ for breathing and talking and of $5.0 \mathrm{~m} \mathrm{~s}^{-1}$ for sneezing and coughing [4], the horizontal distance travelled by SARSCoV-2 for a height of $1.7 \mathrm{~m}$ was $0.88 \mathrm{~m}$ (Fig. 1b) and $2.94 \mathrm{~m}$ (Fig. 1c), respectively, based on Eq. (6).
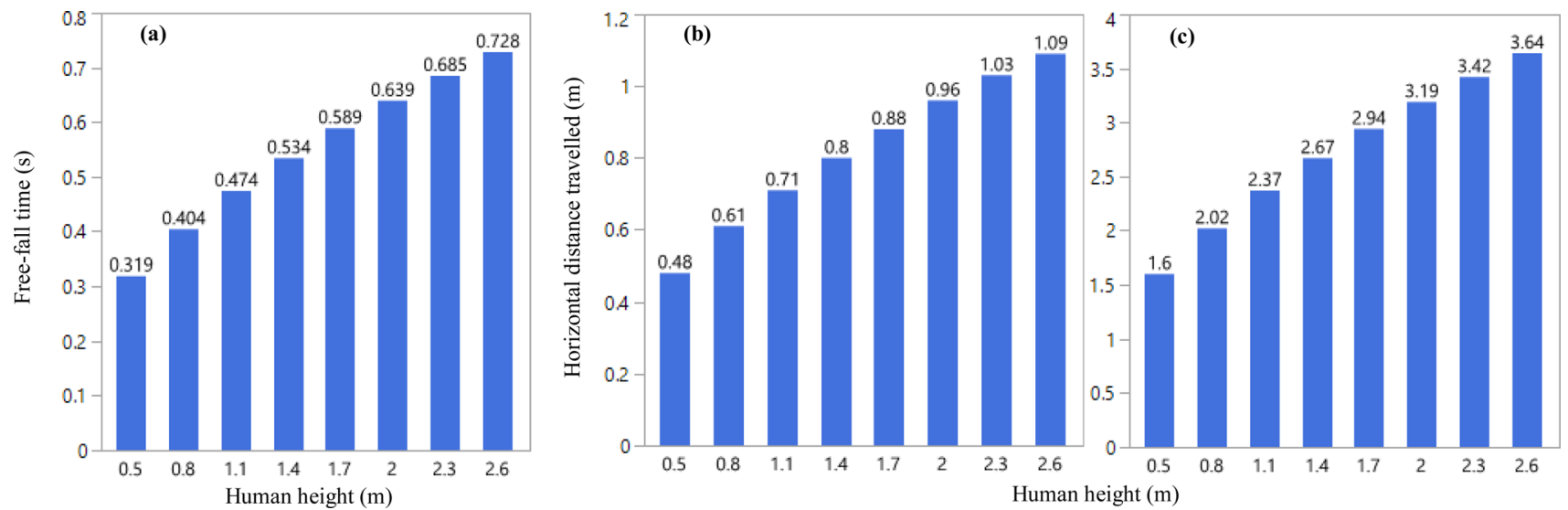

Fig. 1 a Free-fall time of SARS-CoV-2 without a respiratory droplet, and horizontal distance travelled depending on its velocity due to b breathing and talking, and $\mathbf{c}$ sneezing and coughing as a function of human height 


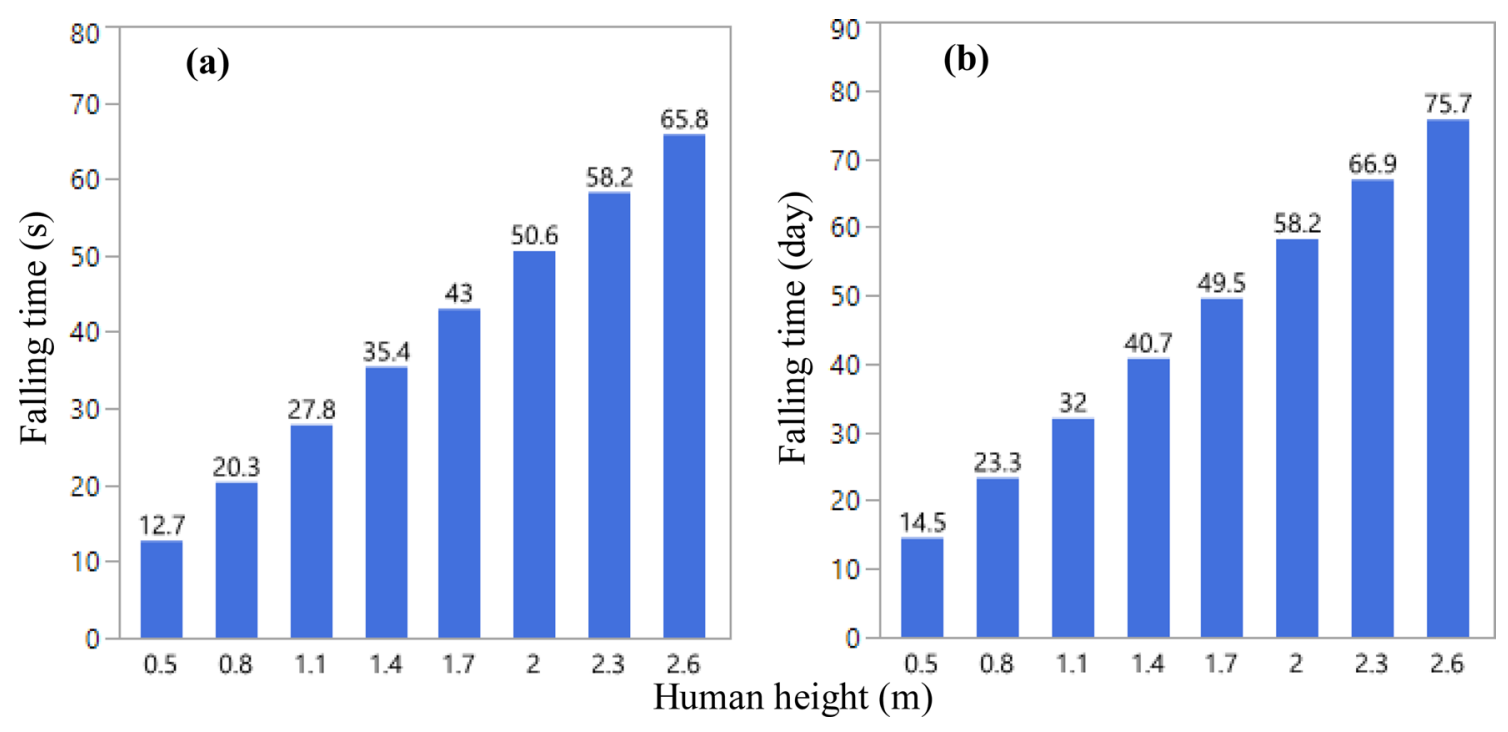

Fig. 2 Falling dynamics of SARS-CoV-2 without a respiratory droplet under the gravity with air resistance according to a Newton's and $\mathbf{b}$ Stokes's laws as a function of human height

According to Eqs. (12) and (13) by Newton's laws of motion, the velocity $\left(v_{t}\right)$ and time $(t)$ of a single SARS-CoV-2 falling from the same human height of $1.7 \mathrm{~m}$ were estimated at $3.95 \times 10^{-2} \mathrm{~m} \mathrm{~s}^{-1}$ and $43 \mathrm{~s}$, respectively (Fig. 2a). In these estimates, the cross-surface area for SARS-CoV-2 where a hemispherical surface area is equal to $2 \pi r^{2}$ was assumed as $1.571 \times 10^{-14} \mathrm{~m}^{2}$. On the contrary, the downward velocity of a single SARS-CoV-2 through the air (the terminal speed: $3.98 \times 10^{-7} \mathrm{~m} \mathrm{~s}^{-1}$ ) is negligible according to Stokes's law. Using Eqs. (18) and (19), a single SARS-CoV-2 from $1.7 \mathrm{~m}$ was estimated to float in the calm air for 49.5 (about 50) days (Fig. 2b). This product of the Stokes's equation means that the gravity does not play a role in its fall. In other words, an isolated SARS-CoV-2 can stay suspended in the calm air for months. However, the estimated duration is not meant to judge its survival duration in the air. In the above estimates, the interactions between SARS-CoV-2 and droplets were not considered. Despite its negligence by the Stokes's equation, the gravity force even for a single virus cannot be denied.

\subsection{The Motion of SARS-CoV-2 with a Respiratory Droplet Under the Gravity with Air Resistance According to Newton's Law}

SARS-CoV-2 is spread through respiratory droplets released from a patient via breathing, talking, coughing, sneezing, or expectorating. Therefore, the velocity and falling time of a single droplet with a single SARS-CoV-2 were estimated as a function of the human height and droplet size using Eqs. (12) and (13), respectively (Fig. 3). Large droplets $>100 \mu \mathrm{m}$, as was classified by Chaudhuri et al. [7], reached the ground from $1.7 \mathrm{~m}$ in a relatively short period of time $<1.6 \mathrm{~s}$. The droplets $\geq 30 \mu \mathrm{m}$ fell within $4.42 \mathrm{~s}$ regardless of the human height (Fig. 3). The droplets with the diameters of 5 and $1 \mu \mathrm{m}$ from $2.6 \mathrm{~m}$ stayed in the calm air for about 11 and $24 \mathrm{~s}$, respectively (Fig. 3).

\subsection{The Motion of SARS-CoV-2 with a Respiratory Droplet According to Stokes's Law}

The outputs of Stokes's law showed a wide range of the falling time of $4.26 \times 10^{-3} \mathrm{~s}\left(4.93 \times 10^{-8}\right.$ days $)$ to $8.83 \times 10^{4} \mathrm{~s}$ (1.02 days) as a function of the droplet size and height (Fig. 4). This was because the falling time of a spherical particle under the gravity in a fluid of a given density and viscosity is inversely proportional to the square of the particle diameter according to Stokes's equation. However, there still exists no simple approach that is likely to be applicable to all the situations and to consider all the relevant factors. Our simulations were independent of evaporation, although the micro-scale droplets may rapidly evaporate under low relative humidity, as was reported by Anchordoqui and Chudnovsky [6], Chaudhuri et al. [7], and Wang et al. [8]. 


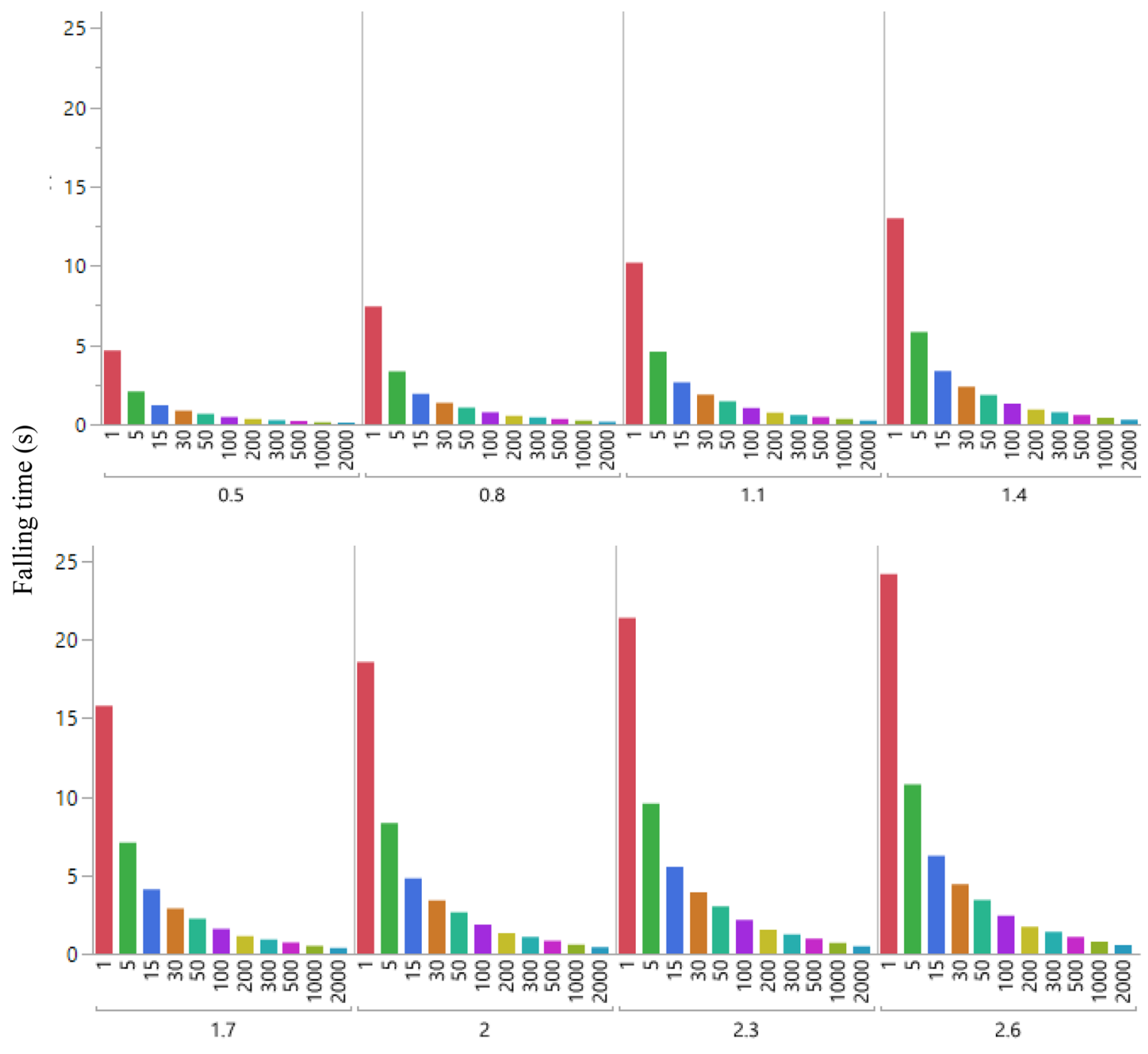

Diameter $(\mu \mathrm{m})$ of a respiratory droplet with SARS-CoV-2, and human height $(\mathrm{m})$

Fig. 3 Falling time of SARS-CoV-2 with a respiratory droplet under the gravity with air resistance according to Newton's law as a function of the droplet diameter and human height

The predictions of the two physical models show a large variation in the falling time of SARS-CoV-2 encapsulated in a respiratory droplet under the gravity with air resistance. The falling time of SARS-CoV-2 with a droplet diameter of $237.5 \mu \mathrm{m}$ remained the same between the two models according to the coefficient value of the best-fit simple linear regression without an intercept (Table 1). The coefficients for the other diameters indicate that the falling times predicted by the two models significantly differed, in particular, in terms of the droplet size $\leq 100$ and $\geq 500 \mu \mathrm{m}$ in diameter.

The spatiotemporal spread dynamics of the COVID19 pandemic is closely coupled to the falling dynamics of
SARS-CoV-2. The first epidemic analysis was introduced in 1927 using a deterministic method called SusceptibleInfected-Recovered (SIR) [9], whose simplest form is described as a non-linear system of ordinary differential equations. The deterministic models of both spread and falling dynamics generate outputs fully determined by their parameter and initial condition values. However, the statistical physics asserts that any deterministic model can be turned into a stochastic model by introducing even a single random variable in the place of some constants through a random resampling from an underlying statistical distribution. Therefore, the falling dynamics predicted by Newton's 

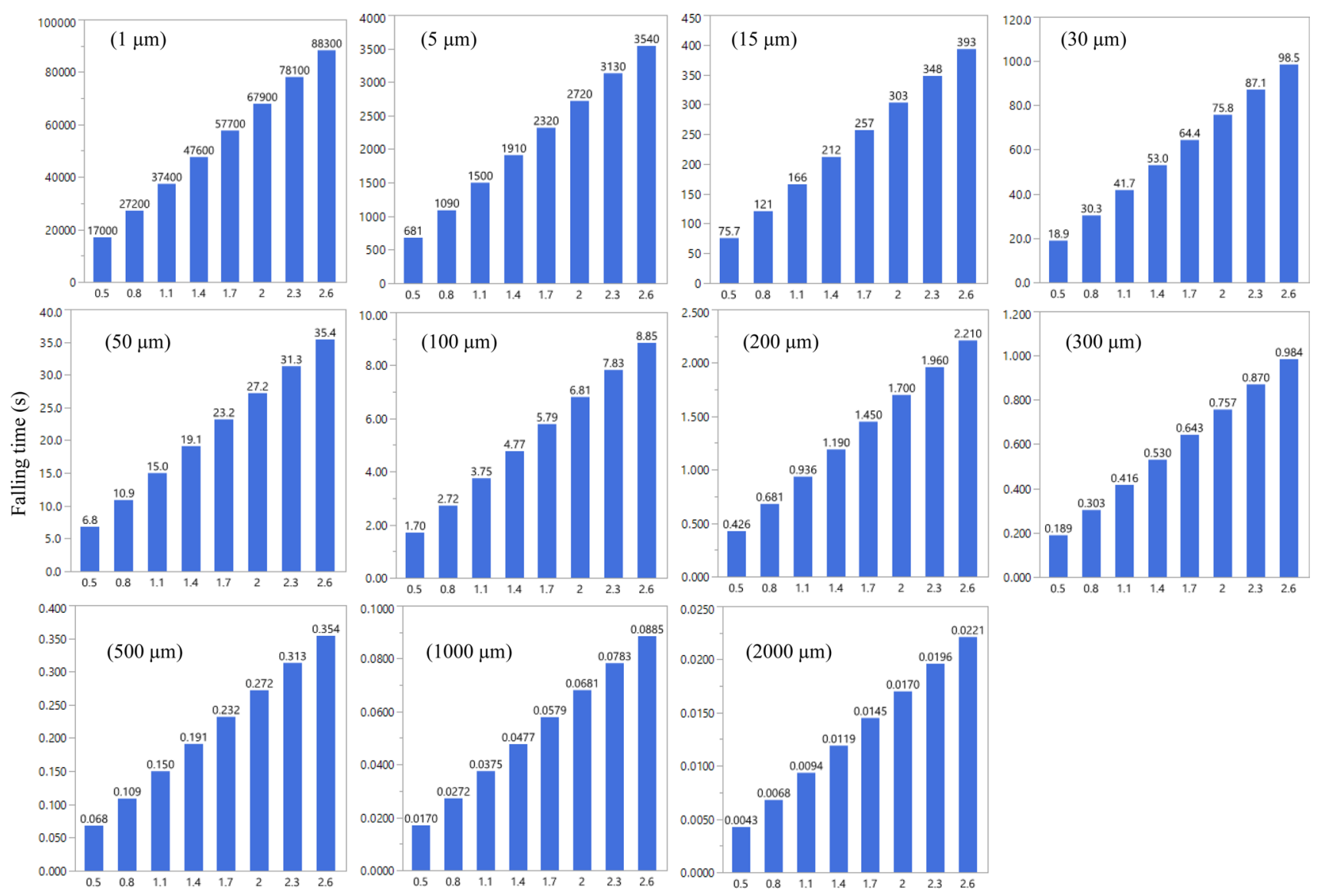

Human height (m)

Fig. 4 Falling time of SARS-CoV-2 with a respiratory droplet under the gravity with air resistance according to Stokes's law as a function of the droplet diameter and human height

Table 1 The best-fit simple linear regression models between the falling times of a respiratory droplet with SARS-CoV-2 estimated by Stokes's $(x)$ and Newton's $(y)$ equations $\left(r^{2}=100 \% ; n=8 ; p<0.05\right)$

\begin{tabular}{llll}
\hline Diameter $(\mu \mathrm{m})$ & $\begin{array}{l}\text { Regression } \\
\text { equation }\end{array}$ & Diameter $(\mu \mathrm{m})$ & $\begin{array}{l}\text { Regression } \\
\text { equation }\end{array}$ \\
\hline 1 & $y=0.0003 x$ & 200 & $y=0.7746 x$ \\
5 & $y=0.0031 x$ & 300 & $y=1.4236 x$ \\
15 & $y=0.0159 x$ & 500 & $y=3.0557 x$ \\
30 & $y=0.0449 x$ & 1000 & $y=8.6529 x$ \\
50 & $y=0.0968 x$ & 2000 & $y=24.489 x$ \\
100 & $y=0.2732 x$ & $\mathbf{2 3 7 . 5}$ & $\boldsymbol{y}=\mathbf{1 . 0 0 0 3 x}$ \\
\hline
\end{tabular}

The droplet diameter shown in bold led to the same falling time regardless of the two models and Stokes's laws as a function of the human height and droplet size were characterized in terms of their statistical distributions. Both were shown to satisfy the lognormal distributions $\left(\mathrm{AIC}_{\mathrm{c}}=353.8\right.$ and 869.8 ; Kolmogorov's D goodness-of-fit test $=0.050$ and $0.075 ; p>0.15$ for Newton's and Stokes's laws, respectively) (Fig. 5). The respiratory droplet diameter of $381.9 \mu \mathrm{m}$, which can barely pass through a perforated cover of 40 mesh in diameter, and the human height of $1.55 \mathrm{~m}$, which can be assumed as the mouth level of a 1.7-m tall patient, were found as the optimal settings to jointly minimize the falling time of SARS-CoV-2. 

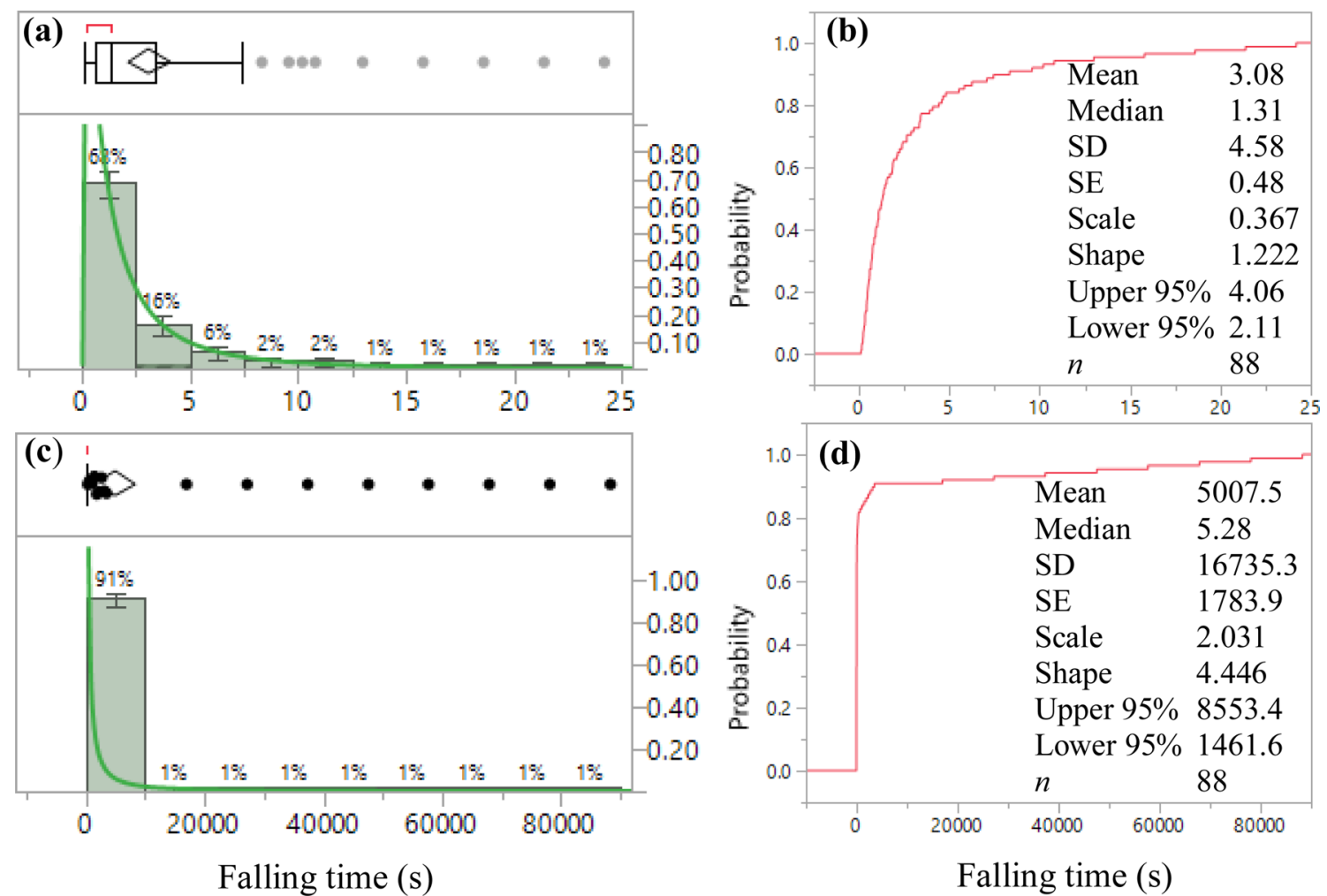

Fig. 5 Lognormal probability density and cumulative distribution functions of the falling times of a respiratory droplet with SARS-CoV-2 according to a-b Newton's and c-d Stokes's laws, respectively

\section{Conclusion}

The estimations in this study without any experimental observations and their validation may not allow for a clearcut conclusion. Newton's and Stokes's laws appeared to be applicable mostly to the droplet size $\geq 237.5 \mu \mathrm{m}$ and $\leq 237.5 \mu \mathrm{m}$, respectively, in better reflecting the motion of SARS-CoV-2. The droplets of 5 and $1 \mu \mathrm{m}$ in diameter released from $1.7 \mathrm{~m}$ stayed in the calm air for $38.7 \mathrm{~min}$ and $16.0 \mathrm{~h}$, respectively, according to the Stokes's equation. Thus, SARS-CoV-2 encapsulated in such respiratory droplets would go airborne for a long time. This might point to the reason for its quick spread around the world. Therefore, an approach still remains to be desired so as to better quantify and predict the motion of the nano-scale objects.

Funding Not applicable.

\section{Compliance with ethical standards}

Conflict of interest All authors declare that they have no conflict of interest.

\section{References}

1. Mushegian, A. R. (2020). Are there $10^{31}$ virus particles on Earth, or more, or less? Journal of Bacteriology, 202(9), e00052-20.

2. Wrapp, D., Wang, N., Corbett, K. S., Goldsmith, J. A., Hsieh, C.-L., Abiona, O., et al. (2020). Cryo-EM structure of the 2019nCoV spike in the prefusion conformation. Science, 367(6483), $1260-1263$.

3. Zhu, N., Zhang, D., Wang, W., Li, X., Yang, B., Song, J., et al. (2020). A novel coronavirus from patients with pneumonia in China, 2019. The New England Journal of Medicine, 382(8), 727-733.

4. Stariolo, D. A. (2020). COVID-19 in air suspensions. arXiv :2004.05699v2.

5. Hillel, D. (1980). Fundamentals of soil physics. New York: Academic Press.

6. Anchordoqui, L. A., \& Chudnovsky, E. M. (2020). A physicist view of the airborne infection. arXiv:2003.13689v1.

7. Chaudhuri, S., Basu, S., Kabi, P., Unni, V. R., \& Saha, A. (2020). Modeling ambient temperature and relative humidity sensitivity of respiratory droplets and their role in Covid-19 outbreaks. arXiv :2004.10929v3.

8. Wang, B., Zhang, A., Sun, J. L., Liu, H., Hu, J., \& Xu, L. X. (2005). Study of SARS transmission via liquid droplets in air. Journal of Biomechanical Engineering, 127, 32-38.

9. Kermack, W. O., \& McKendrick, A. G. (1927). A contribution to the mathematical theory of epidemics. Proceedings of the Royal Society of London A: Mathematical, Physical and Engineering Sciences, 115, 700-721. 